\section{Revista 2019 \\ Ciencia e Ingeniería Vol. 29(1) \\ Neogranadina Enero-junio 2019}

DOI: https://doi.org/10.18359/rcin.3529

\title{
Caracterización, procesabilidad y viabilidad técnica de fondos de taza de caucho natural
}

\author{
Natalia Cardona-Vásquez ${ }^{\mathbf{a}}$ - Diego Hernán Giraldo-Vásquez ${ }^{\mathbf{b}}$ \\ - Sandra Milena Velásquez-Restrepo`
}

\begin{abstract}
Resumen: En este artículo, se compararon propiedades fisicoquímicas, espectroscópicas, de resistencia a la termooxidación y de procesabilidad de fondos de taza de caucho natural de los clones IAN 873 y FX 3864 respecto del caucho natural técnicamente especificado grado 10 proveniente de Guatemala, uno de los más importados en Colombia y en América Latina. Se determinaron también sus características de procesamiento en reómetro de torque asociadas al consumo de energía. Se elaboraron compuestos con un sistema de vulcanización semieficiente y se evaluaron propiedades reométricas y los parámetros de vulcanización a $160^{\circ} \mathrm{C}$ mediante reometría de disco móvil, con los que se efectuó la vulcanización de probetas estandarizadas para la evaluación de propiedades en tensión y dureza Shore A. Se encontró que los fondos de taza presentaron un mayor contenido de gel, lo que tuvo un efecto en la reacción de vulcanización de los compuestos; no obstante, no se encontraron diferencias significativas en las características de procesamiento en mezclador interno ni en las propiedades tecnológicas de los compuestos vulcanizados. Estos resultados muestran que los fondos de taza representan una alternativa técnicamente viable para la fabricación de productos en caucho natural.
\end{abstract}

Palabras clave: fondos de taza; caucho técnicamente especificado; fase gel; propiedades tecnológicas.

Fecha de recepción: 18 de junio de 2018 Fecha de aprobación: 28 de agosto de 2018

Cómo citar: N. Cardona-Vásquez, D. H. Giraldo-Vásquez y S. M. Velásquez-Restrepo, "Caracterización, procesabilidad y viabilidad técnica de fondos de taza de caucho natural", Ciencia e Ingeniería Neogranadina, vol. 29(1), pp. 37-52. https://doi.org/10.18359/rcin.3529

a Universidad de Antioquia, Colombia.e-mail: natalia.cardonav@udea.edu.co. ORCID: https://orcid.org/0000-0002-3348-0261

b Universidad de Antioquia, Colombia. e-mail: dhernan.giraldo@udea.edu.co. ORCID: https://orcid.org/0000-0002-4306-9978

c Servicio Nacional de Aprendizaje, Colombia. e-mail: smvelasquez@sena.edu.co. ORCID: https://orcid.org/0000-0002-6697-2801 


\title{
Characterization, Processability, and Technical Viability of Natural Rubber Cup Lumps
}

\begin{abstract}
This study compares the physicochemical and spectroscopic characteristics, resistance to thermooxidation, and processability of natural rubber cup lumps of clones IAN 873 and FX 3864 with those of technically specified grade 10 natural rubber from Guatemala - one of the most commonly imported rubbers in Colombia and Latin America. Additionally, processing characteristics associated with energy consumption were determined through torque rheometry. Therefore, compounds were formed using a semi-efficient vulcanization system. Further, rheometric properties and vulcanization parameters were measured at $160{ }^{\circ} \mathrm{C}$ in a moving die rheometer. Moreover, these measurements were used to vulcanize the standardized samples and to test stress and hardness properties based on the Shore A scale. The cup lumps were found to have a higher gel content, which affected the vulcanization reaction of the compounds; however, significant differences were found neither in internal mixer processing characteristics nor in the technological properties of the vulcanized compounds. These results reveal that cup lumps represent a technically viable alternative for the production of natural rubber products.
\end{abstract}

Keywords: Cup Lumps; Technically Specified Rubber; Gel Phase; Technological Properties.

\section{Caracterização, processabilidade e viabilidade técnica de borracha natural do fundo de recipiente}

Resumo: O presente estudo compara as características físicoquímicas, espectroscópicas, de resistência a termo-oxidação e a processabilidade de borracha natural do fundo de recipiente dos clones IAN 873 e FX 3864 com especificação técnica de grau 10 de borracha natural proveniente da Guatemala, sendo uma das borrachas mais importada na Colômbia e América Latina. Foi determinado também suas características de processamento com um reômetro de torque associado ao consumo de energia. Foram elaborados compostos com um sistema de vulcanização semi-eficiente e foram avaliadas as propriedades reométricas e os parâmetros de vulcanização a $160^{\circ} \mathrm{C}$, através de reometria de disco móvel, com os quais foram efetuadas as vulcanizações de corpos de prova padronizados para a avaliação de propriedades de tensão e dureza conforme a escala Shore A. Foi descoberto que os fundos de recipiente apresentaram um maior conteúdo de gel, o qual teve um efeito sobre a reação de vulcanização dos compostos. Não obstante, não foi encontrado diferenças significativas nas características de processamento no misturador interno e nem nas propriedades tecnológicas dos compostos vulcanizados. Estes resultados mostram que os fundos de recipeinte representam uma alternativa tecnicamente viável para a fabricação de produtos em borracha natural.

Palavras chave: Fundos de recipiente; borracha tecnicamente especificado; fase gel; propriedades tecnológicas. 


\section{Introducción}

El caucho natural es una materia prima de alta demanda mundial, ya que es fundamental para la fabricación de productos usados en diversos sectores económicos [1].

Colombia, como país con un amplio potencial agrícola, ha venido apostando en los últimos años al aumento del número de hectáreas sembradas de caucho natural, con miras a impulsar la explotación de este recurso natural que ha mostrado una buena adaptación en algunas regiones [2]. Según el último censo cauchero nacional, Colombia cuenta con aproximadamente 54000 ha sembradas de caucho natural, pero solo el $5 \%$ se encuentran en la etapa productiva, por lo que el país importa cerca del 95\% del material que consume [3]. Además, cerca del 98\% de los heveicultores son pequeños productores, lo que ha dificultado la capacitación y estandarización de los procesos productivos de manera tal que se garantice una mayor calidad y baja variabilidad en las propiedades de los materiales obtenidos [4]. En países como Brasil, Ecuador y Guatemala, el papel de los pequeños productores es similar.

Los fondos de taza de caucho natural corresponden al material coagulado de manera natural o inducida a través de la adición de un ácido a la suspensión de látex. A pesar de ser empleados para la fabricación de caucho técnicamente especificado, los fondos de taza no tienen un alto valor comercial, debido al desconocimiento de sus propiedades y por la variabilidad que estas presentan [5]. Uno de los factores que más influye en las diferencias de las características de este material es la diversidad de tamaños en los que pueden obtenerse, ya que la productividad de cada árbol independiente de su variedad clonal varía de acuerdo con la adaptación y respuesta de cada individuo a las condiciones edafoclimáticas del entorno donde se desarrolla y la etapa fenológica en la que se encuentra [5]. Otro de los factores de gran influencia es la formación de la fase gel, que corresponde a la interacción de los lípidos y de las proteínas que se encuentran en las terminales de la cadena polimérica del caucho natural; esta interacción se da de manera aleatoria durante el proceso de coagulación, por tanto, no es posible controlar efectivamente su formación [6].

En este trabajo, se emplearon análisis fisicoquímicos, espectroscópicos y de procesamiento, para caracterizar fondos de taza de caucho natural de los clones IAN 873 y FX 3864 provenientes de una pequeña plantación ubicada en el municipio de Maceo, departamento de Antioquia, así como para el caucho técnicamente especificado grado 10 de Guatemala (SGR 10). Se realizaron mezclas estándar con un sistema de vulcanización semieficiente, y se evaluaron propiedades en tensión y la dureza de los compuestos luego de vulcanizados. Los resultados obtenidos permitieron comparar las mezclas elaboradas a partir de fondos de taza con las del SGR 10, una de las materias primas más importadas en Colombia y muchos países latinoamericanos. Además, se evaluó la viabilidad técnica de usar fondos de taza como insumo para la fabricación de productos en caucho natural.

\section{Materiales y métodos de ensayo}

\section{Materiales}

Los fondos de taza fueron recolectados de árboles de caucho natural de los clones IAN 873 y FX 3864 provenientes de un cultivo ubicado en Maceo, departamento de Antioquia, en la etapa fenológica de foliación. En el momento de la recolección efectuada, el cultivo tenía una edad productiva de 2 meses. Se determinó la cantidad de fondos de taza por recolectar de acuerdo con el número de árboles pertenecientes a cada variedad clonal, por lo que se seleccionó una muestra representativa de cada población empleando las ecuaciones (1) y (2). Se realizaron dos muestreos para la obtención de la materia prima. Estas condiciones de recolección fueron definidas en busca de bloquear la variedad clonal y el periodo fenológico como fuentes de variabilidad que han sido ampliamente reportadas [2].

$$
n^{\prime}=\frac{n_{0}}{1+\left[\frac{n_{0}-1}{N}\right]}
$$


Donde:

n’: número de muestra ajustado

$\mathrm{n}_{0}$ : número de muestra teórico

$\mathrm{N}$ : tamaño de la población

$n_{0}=\frac{\left[z^{2}\right][p][q]}{e^{2}}$

Donde:

z: nivel de confianza

p: probabilidad de que la muestra sea representativa de la población

q: probabilidad de que la muestra no sea representativa de la población

[p][q]: varianza

$\mathrm{e}^{2}$ : error máximo permitido

Todos los fondos de taza fueron obtenidos mediante coagulación en recipientes de igual volumen y fabricados con el mismo material. Posterior a su recolección, fueron secados a la sombra en el cultivo de estudio durante 20 días y luego fueron trasladados a Medellín, donde fueron lavados con agua corriente para remover impurezas (tierra, chamizos, insectos, entre otros) de la superficie. Por último, se dejaron secando a la sombra durante 20 días más, sin exposición a luz solar y con circulación de aire constante.

El caucho técnicamente especificado grado 10 de Guatemala (SGR 10) empleado para todo el análisis fue tomado de una misma bala 1 de material (1 corresponde a $33,33 \mathrm{~kg}$ de material compactado, con dimensiones de $70 \mathrm{~cm} \times 35 \mathrm{~cm} \times 18 \mathrm{~cm}$ ). Este material cuenta con unas especificaciones técnicas de acuerdo con lo estipulado en la norma ASTM D2227, y presenta valores máximos para el contenido de nitrógeno, cenizas e índice de retención de plasticidad, que no son comúnmente determinadas para los fondos de taza.

\section{Métodos de ensayo}

\section{Determinación del contenido de nitrógeno, cenizas y metales}

Se determinó el contenido de nitrógeno para fondos de taza de cada clon, mediante el método Kjeldahl de acuerdo con la NTC 370. El contenido de cenizas se determinó mediante gravimetría de acuerdo con la NTC 5167. Los contenidos de calcio, magnesio, potasio y sodio fueron determinados mediante electroforesis capilar y el hierro mediante absorbancia atómica según la SM 3111B. Todos los parámetros fueron comparados con los valores estipulados en la norma ASTM D2227 para el caucho técnicamente especificado grado 10. Para todos los materiales de estudio, se evaluó una muestra.

\section{Determinación del contenido de gel}

Para el cálculo del contenido de gel, se siguieron las recomendaciones de la norma ISO 17278:2013. Para los fondos de taza se efectuaron mediciones en el núcleo y el exterior de los fondos de taza, tal como se muestra en la figura 1 . Se efectuaron seis réplicas por material.

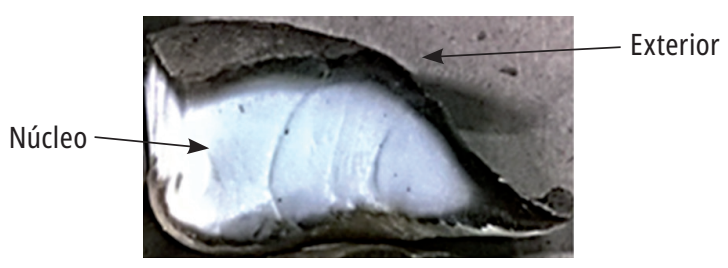

Figura 1. Muestra obtenida luego de efectuar un corte transversal a un fondo de taza de caucho natural. Fuente: Elaboración propia.

\section{Determinación de la resistencia a la termooxidación}

Se realizaron ensayos de índice de retención de plasticidad (PRI, por sus siglas en inglés), según lo estipulado en la norma ASTM D3194. Se empleó un plastímetro Wallace de platos paralelos marca CG Technology, aplicando una fuerza compresiva de $100 \mathrm{~N}$ y una temperatura de $100^{\circ} \mathrm{C}$. En los fondos de taza, se efectuó la medición para materiales recolectados en los dos muestreos realizados. Para determinar el valor de PRI, se empleó la relación especificada en la ecuación (3).

$$
P R I=\left[\frac{P_{1}}{P_{0}}\right] * 100
$$

Donde:

$\mathrm{P}_{0}$ : plasticidad de la muestra sin envejecer

$\mathrm{P}_{1}$ : plasticidad de la muestra envejecida 


\section{Análisis de bandas y grupos funcionales}

Este análisis se efectuó mediante espectroscopia infrarroja por transformada de Fourier con reflectancia total atenuada (FTIR-ATR), en un equipo IRTracer-100, empleando el modo de absorbancia en un rango de número de onda de entre 750 y $4000 \mathrm{~cm}^{-1}$, una resolución de $2 \mathrm{~cm}^{-1}$, un porcentaje de corrección de atmósfera del 1,5\% y un 9\% de suavizado. La corrección de línea base para todos los espectros obtenidos se efectuó según 15 puntos fijos tomando puntos característicos asociados al caucho natural; estos puntos tenían el mismo número de onda asociado en el momento de la corrección.

Todos los espectros fueron normalizados respecto de un pico invariable $\left(835 \mathrm{~cm}^{-1}\right)$ para todas las muestras, con el fin de poder comparar los resultados obtenidos, dado que se identificó como el pico invariante entre espectros. Este pico es el asociado a la flexión fuera del plano del enlace $=\mathrm{C}-\mathrm{H}$, grupo funcional característico de la molécula del cis 1,4 poliisopreno.

\section{Características de procesamiento en reómetro de torque (mezclador interno)}

Los ensayos se realizaron en un reómetro de torque HAAKE PolyLab OS con rotores tipo Banbury. Se evaluaron tres réplicas por material, aplicando un factor de llenado del $70 \%$ y una temperatura inicial de $60^{\circ} \mathrm{C}$. Se cortaron trozos de material con geometría similar para facilitar la alimentación por la tolva y el posterior procesamiento en busca de disminuir la variabilidad en los datos obtenidos. Se determinó el torque final, la temperatura final y el tiempo de masticación en la zona efectiva de masticación, correspondiente al periodo entre el cierre del pistón y la estabilización del torque.

El consumo de potencia durante este método de procesamiento fue calculado relacionando únicamente la curva asociada al torque aplicado durante el periodo de masticación de acuerdo con la ecuación (4) [7]. Esta medición se incluyó como característica de procesamiento de los cauchos naturales estudiados.
$P=\left[\frac{\frac{2 \cdot \pi \cdot N}{m} \int M . d t}{t}\right]$

Donde:

P: potencia $(\mathrm{kW} / \mathrm{kg})$

$\mathrm{N}$ : velocidad de los rotores (rpm)

M: torque registrado por el reómetro (N.m)

$\mathrm{m}$ : masa de la muestra $(\mathrm{kg})$

$\mathrm{t}: \quad$ tiempo (min)

$\int M . d t$ : área bajo la curva de torque versus tiempo

\section{Elaboración de mezclas}

Se diseñaron compuestos de 250 g según una formulación de sistema semieficiente tomando como base lo estipulado en la norma ASTM D3184. La masa empleada fue seleccionada para garantizar la formación de banco durante la elaboración de las mezclas en un molino abierto de rodillos marca Bolling de 15,24 cm de diámetro por $30,48 \mathrm{~cm}$ de largo, con una relación de giro entre rodillos de 1:1,25.

En la tabla 1, se presenta la formulación empleada, en la que se indica la proporción de cada ingrediente como partes en peso por cada 100 partes de caucho (phr, por sus siglas en inglés), nomenclatura universalmente empleada en la literatura científica y técnica para formulaciones de caucho.

\section{Reometría de vulcanización MDR}

Los ensayos fueron realizados a $160^{\circ} \mathrm{C}$ basados en las recomendaciones de la ASTM D5289, empleando un reómetro marca Pioneer de Alpha

Tabla 1. Formulación semieficiente empleada para la elaboración de los compuestos

\begin{tabular}{l|c}
\multicolumn{1}{c|}{ Elemento } & Partes en peso (phr) \\
Caucho natural & 100,0 \\
\hline Óxido de zinc & 5,0 \\
\hline Ácido esteárico & 2,0 \\
\hline Azufre & 1,35 \\
\hline TBBS* & 1,85 \\
\hline Proporción TBBS/azufre & 1,37 \\
\hline * N-tert-butil-2-benzotiazol sulfenamida. \\
Fuente: Elaboración propia.
\end{tabular}


Technologies. Para cada material, se evaluaron tres réplicas. Los parámetros obtenidos para cada compuesto se definen a continuación:

- Tiempo de inicio de vulcanización o scorch $\left(t_{\mathrm{s}}\right)$ : es el tiempo requerido para que inicie la reacción de vulcanización.

- Tiempo de vulcanización completa $\left(\mathrm{t}_{100}\right)$ : es el tiempo requerido para que se complete la reacción de vulcanización.

- Torque mínimo (ML): es el torque asociado a la rigidez elástica mínima del material no vulcanizado.

- Torque máximo (MH): es el torque máximo asociado a la rigidez elástica del material al completar totalmente la reacción de vulcanización.

\section{Vulcanización de probetas de tensión}

Todas las probetas para la evaluación de las propiedades en tensión fueron obtenidas con la geometría tipo IV especificada en la norma ASTM D412, mediante moldeo por compresión a $160^{\circ} \mathrm{C}$ según el tiempo de vulcanización completa $\left(t_{100}\right)$ obtenido de la reometría de vulcanización MDR. Se empleó una prensa marca Pinzuar con una presión máxima de 3000 psi. Para cada material, se vulcanizó una lámina de $150 \mathrm{~mm} \times 150 \mathrm{~mm} \times 2$ $\mathrm{mm}$ de espesor.

\section{Evaluación de propiedades mecánicas en tensión y dureza}

Se evaluó la resistencia a la tensión, módulo al 100 y al 300\% de deformación unitaria, y la elongación a la rotura, aplicando una velocidad de $500 \mathrm{~mm} / \mathrm{min}$ de acuerdo con lo estipulado en la norma ASTM D412. Se evaluó la dureza Shore A basados en lo estipulado en el método manual de la norma ASTM D2240 en una zona cercana al área de observación de las probetas de tensión. Debido a que el espesor de una probeta no abarcaba el mínimo requerido para la medición según lo especificado en la norma, se tomaron varias probetas y se superpusieron hasta alcanzar un espesor de al menos $6 \mathrm{~mm}$. Se evaluaron cinco réplicas por cada material vulcanizado.

\section{Resultados y discusión}

\section{Contenido de nitrógeno, cenizas y metales}

En la tabla 2, se presentan los resultados obtenidos para los fondos de taza de los clones IAN 873, FX 3864 y para el material de referencia SGR 10. Además, se comparan con los valores estipulados en la norma ASTM D2227 para el caucho natural técnicamente especificado grado 10 .

En la tabla 2, se observa que, a pesar de que los fondos de taza no fueron sometidos a procesos de estandarización, tienen un contenido de cenizas inferior al máximo estipulado por la norma ASTM D2227; en el caso del contenido de nitrógeno, se aprecia que el clon IAN 873 presenta un contenido inferior incluso al obtenido para el SGR 10, mientras que el FX 3864 exhibió un valor levemente superior. Estos resultados demuestran una variabilidad en esta característica fisicoquímica atribuible al tipo de clon.

Los resultados de los análisis fisicoquímicos muestran que todos los materiales se encuentran en el rango estipulado en la norma ASTM D2227, por tanto, se consideran aptos para la realización

Tabla 2. Comparación del contenido de cenizas, nitrógeno orgánico, hierro y cobre para fondos de taza de los clones IAN 873, FX 3864 y el material de referencia SGR 10.

\begin{tabular}{l|c|c|c|c}
\hline Parámetro & ASTM D2227 & SGR 10 & Fondos de taza clon IAN 873 & Fondos de taza clon FX 3864 \\
\cline { 5 - 6 } Cenizas (\%) & 0,75 máx. & $0,35 \pm 0,06$ & $0,47 \pm 0,11$ & $0,47 \pm 0,09$ \\
\hline Nitrógeno orgánico total (\%) & 0,60 máx. & 0,39 & 0,16 & 0,64 \\
\hline Hierro (Fe) (ppm) & - & $\leq 0,100$ & $\leq 0,100$ & $\leq 0,100$ \\
\hline Cobre $(\mathrm{Cu})(\mathrm{ppm})$ & - & $\leq 0,100$ & $\leq 0,100$ & $\leq 0,100$ \\
\hline
\end{tabular}

Fuente: Elaboración propia. 
de compuestos de caucho natural. No obstante, es necesario contrastar con resultados posteriores para establecer si las diferencias encontradas respecto del contenido de nitrógeno influyen en sus propiedades finales.

\section{Contenido de gel}

En la figura 2, se presentan diagramas de caja para el contenido de gel de las muestras tomadas de la zona del núcleo y el exterior de fondos de taza de los clones IAN 873 y FX 3864, así como para el SGR 10 como material de referencia. Estos diagramas muestran los cuartiles 25 y $75 \%$, así como los valores mínimos y máximos. Al efectuar un análisis de variancia (ANOVA, por sus siglas en inglés) con un 0,05 de significancia, fue posible establecer que no existe una diferencia estadísticamente significativa entre las muestras tomadas del núcleo y el SGR 10. Se encontró una marcada diferencia entre el contenido de gel de las muestras tomadas del núcleo de los fondos de taza respecto del exterior y el SGR 10. Asimismo, no se encontraron diferencias significativas atribuibles al muestreo, lo que se atribuye al hecho de haber tomado las muestras dentro del mismo periodo fenológico que bloqueó una fuente de variación adicional.

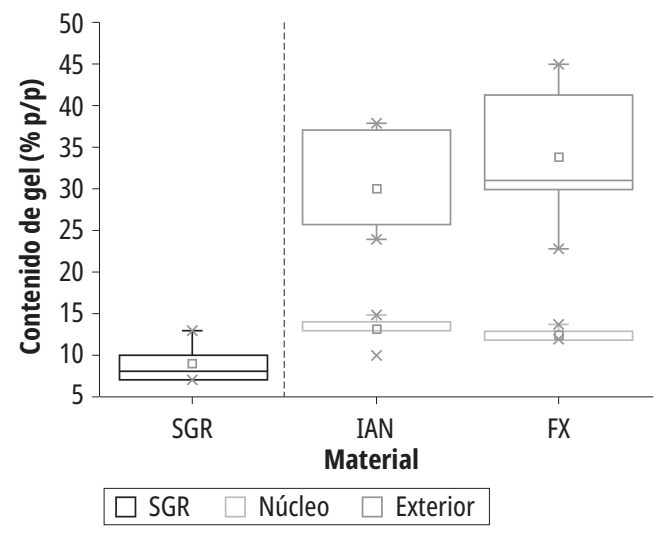

Figura 2. Contenido de gel de muestras tomadas del material de referencia y del núcleo y exterior de los fondos de taza de los clones IAN 873 y FX 3864. Fuente: Elaboración propia.

La diferencia existente entre el núcleo y el exterior de los fondos de taza indica que la exposición al medio ambiente favoreció el aumento del contenido de gel en el exterior. Esto se explica debido a que los fondos de taza durante su proceso de coagulación interactúan con el aire y generan una oxidación de las cadenas poliméricas, un fenómeno que es favorecido por la temperatura $[6,8]$, lo que facilita una mayor formación de la fase gel en el material [9]. De igual manera, debe tenerse presente que la exposición al medio ambiente produce un aumento en la variabilidad del contenido de gel, ya que originalmente la distribución de las cadenas de poliisopreno dentro de la partícula de caucho seca no es homogénea [10], y cuando dichas cadenas se encuentran involucradas en procesos oxidativos, estos también se producen de manera no uniforme.

Tales resultados demuestran que los fondos de taza presentan variabilidad incluso dentro de una misma muestra, por lo que es necesario analizarlos en conjunto con la determinación de la resistencia a la termooxidación, los parámetros de vulcanización y las propiedades mecánicas, y así establecer si los fondos de taza son aptos para la elaboración de productos en caucho sin someterlos a procesos previos de estandarización.

\section{Resistencia a la termooxidación}

En la figura 3, se presentan los resultados del PRI obtenidos para los fondos de taza de las muestras tomadas (1 y 2) de los clones IAN 873 y FX 3864, así como para el SGR 10 como material de referencia.

En la figura 3, se observa que el PRI de las muestras tomadas de los fondos de taza de los clones IAN 873 y FX 3864 presentan valores superiores al del SGR 10, lo que indica que ambos tipos de fondos de taza exhiben una mayor resistencia a la termooxidación que el material de referencia de acuerdo con los rangos especificados en la norma ASTM D3194 [11]. Debe considerarse que, durante la elaboración de caucho natural técnicamente especificado, el material es sometido a temperaturas de hasta $140^{\circ} \mathrm{C}$, a la cual ya se han iniciado procesos de termooxidación interna en las cadenas poliméricas [11]. No obstante, el caucho SGR 10 evaluado en este estudio presenta un valor del PRI superior al mínimo estipulado en la norma ASTM D2227 (50\% mínimo). 


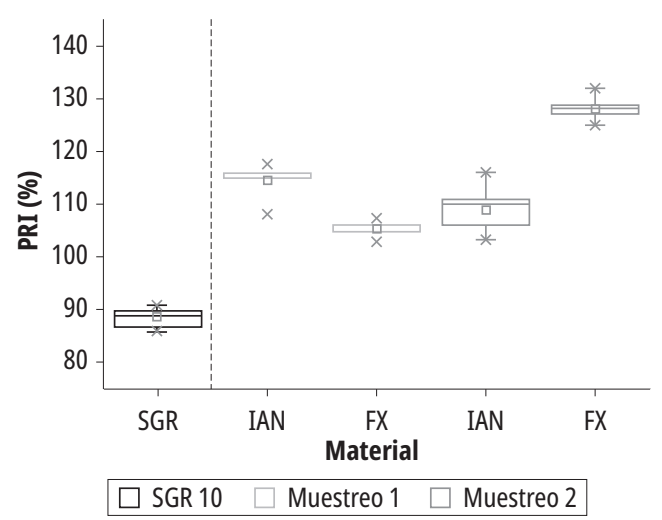

Figura 3. Valores del PRI para los fondos de taza de caucho natural de los clones IAN 873 y FX 3864 y para el material de referencia SGR 10. Fuente: Elaboración propia.

Por otra parte, los ANOVA con 0,05 de significancia efectuados permitieron establecer que existen diferencias en el valor del PRI atribuible al muestreo. Se han reportado estudios en los que se ha logrado concluir que durante los primeros 300 días de rayado el caucho natural obtenido de los árboles presenta un valor de PRI variable que tiende a estabilizarse después de este periodo [12], lo que concuerda con lo hallado en este trabajo, pues la edad productiva del cultivo en el momento de efectuar la recolección de las muestras era de dos meses. En la diferencia presentada entre clones, debe tenerse presente que cada variedad clonal responde de manera diferente a las condiciones edafoclimáticas y esto puede influir de manera significativa en su comportamiento frente a fenómenos de termooxidación [13].

\section{Análisis de bandas y grupos funcionales}

En la figura 4, se presentan espectros normalizados representativos obtenidos mediante FTIRATR, tomados de muestras del núcleo y del exterior de los fondos de taza, así como para el SGR 10 como material de referencia. Se señalan con flechas las zonas en las que se observó mayor diferencia entre los espectros obtenidos para las muestras analizadas.

En la figura 4, es posible observar que existen diferencias entre los espectros de las muestras tomadas del núcleo y el exterior de los fondos de taza; sin embargo, al comparar las muestras tomadas del exterior con el SGR 10, no se observan diferencias entre los espectros obtenidos. Las bandas de absorción asociadas a sustancias proteicas están estipuladas entre 3300 y $3100 \mathrm{~cm}^{-1}$, donde precisamente se encontró la diferencia más marcada para las muestras evaluadas del núcleo de los dos clones y el SGR 10. Estos picos se atribuyen a la vibración de la banda $\mathrm{V}-\mathrm{N}-\mathrm{H}$; las proteínas también están asociadas a los picos observados a 1630 y $1541 \mathrm{~cm}^{-1}$, pertenecientes a los grupos Amida I $(V-(C=O)-N H)$ y Amida II $(\beta-\mathrm{N}-\mathrm{H}+V-C-N)$, respectivamente. Los picos presentes en las regiones próximas a 2928 y $2851 \mathrm{~cm}^{-1}$ corresponden a los estiramientos simétricos y asimétricos del radical metilo ( $\mathrm{V}$-sim. $\mathrm{C}-\mathrm{H}_{2^{-}} \mathrm{y} \mathrm{V}$-asim $\mathrm{C}-\mathrm{H}_{2^{-}}$), mientras que en $2960 \mathrm{~cm}^{-1}$ se presenta el estiramiento asimétrico del C-H en el radical metilo ( $V$-asim. $\mathrm{C}-\mathrm{H}_{3}$ ). La presencia de lípidos se evidencia con los picos comprendidos en $1748-1738 \mathrm{~cm}^{-1}$ y el observado a $1710 \mathrm{~cm}^{-1}$, correspondientes a los estiramientos del grupo éster y carboxilo $(V-(\mathrm{C}=\mathrm{O})-\mathrm{O}-; \mathrm{V}-(\mathrm{C}=\mathrm{O})$ $\mathrm{OH})$, respectivamente. Por último, el pico observado a $835 \mathrm{~cm}^{-1}$ está asociado a la flexión del -C-H- fuera del plano en el grupo funcional $\mathrm{C}=\mathrm{C}$, que es característico para los enlaces asociados a la molécula del cis 1,4 poliisopreno [14].

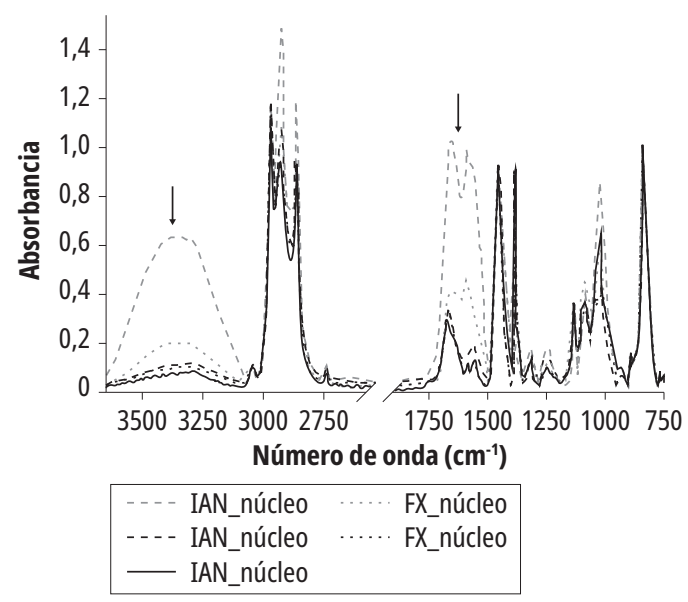

Figura 4. Espectros obtenidos mediante FTIR-ATR de muestras tomadas del núcleo y exterior de los fondos de taza y para el material de referencia SGR 10. Fuente: Elaboración propia. 
Las diferencias encontradas entre las muestras tomadas del núcleo y el exterior de los fondos de taza indican que el material tomado del núcleo presenta una mayor concentración de proteínas. Si bien las bandas en las regiones de $3284 \mathrm{~cm}^{-1} \mathrm{y}$ de $3332 \mathrm{~cm}^{-1}$ también pueden obedecer a los ácidos orgánicos o humedad [15], al no encontrar en la figura 4 bandas representativas entre 1630 y 1541 $\mathrm{cm}^{-1}$, es posible establecer que sí existen diferencias entre el núcleo y el exterior de los fondos de taza.

Es importante resaltar que durante el procesamiento del caucho natural las muestras son sometidas a procesos de masticación, durante los que se involucran procesos termomecánicos y termooxidativos; en ese punto, ya el material del núcleo se incorpora dentro de todo el material proceso y su color cambia de blanco a café, es decir, adquiere la misma apariencia del exterior. En este sentido, es necesario observar el comportamiento de los materiales de interés durante el procesamiento en reómetro de torque y si existen diferencias en los parámetros de vulcanización y propiedades mecánicas que puedan ser relacionadas con lo encontrado en el contenido de gel y el análisis mediante FTIR-ATR.

\section{Características de procesamiento en reómetro de torque (mezclador interno)}

Las características de procesamiento en mezclador interno fueron determinadas desde el cierre de la cámara hasta el tiempo en que el torque fue estable.
Los parámetros de procesamiento por determinar para la realización de la comparación entre los materiales se esquematizan en la curva representativa mostrada en la figura 5 .

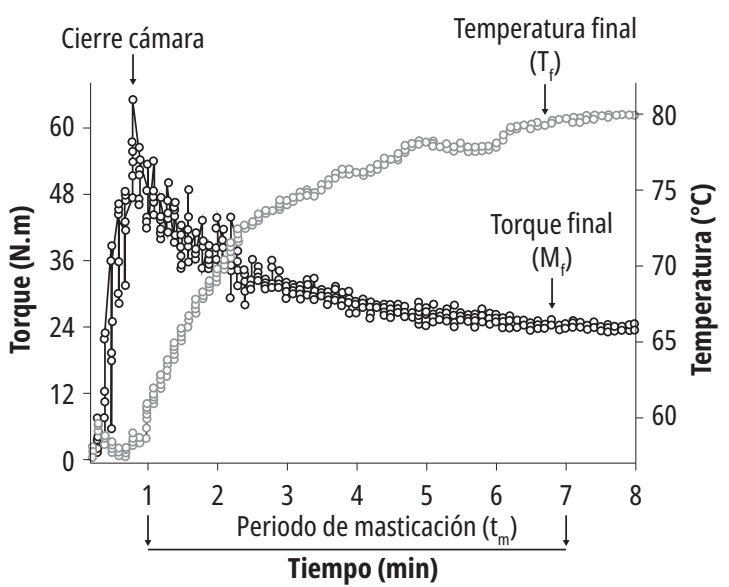

Figura 5. Curva representativa de torque (N.m) versus tiempo (min). Fuente: Elaboración propia.

En la figura 6, se presentan diagramas de caja para los valores de torque final $\left(\mathrm{M}_{\mathrm{f}}\right)$, periodo de masticación $\left(\mathrm{t}_{\mathrm{m}}\right)$, temperatura final $\left(\mathrm{T}_{\mathrm{f}}\right)$ y potencia (P). Se observa que para todos los parámetros las muestras que presentan mayor variabilidad son las procesadas de los fondos de taza. A pesar de que, al efectuar el ANOVA con 0,05 de significancia fue posible establecer que no existen diferencias significativas entre las muestras evaluadas para estos parámetros, la comparación indica que el material de referencia SGR 10 requiere más tiempo para alcanzar la estabilización del torque, así como que el
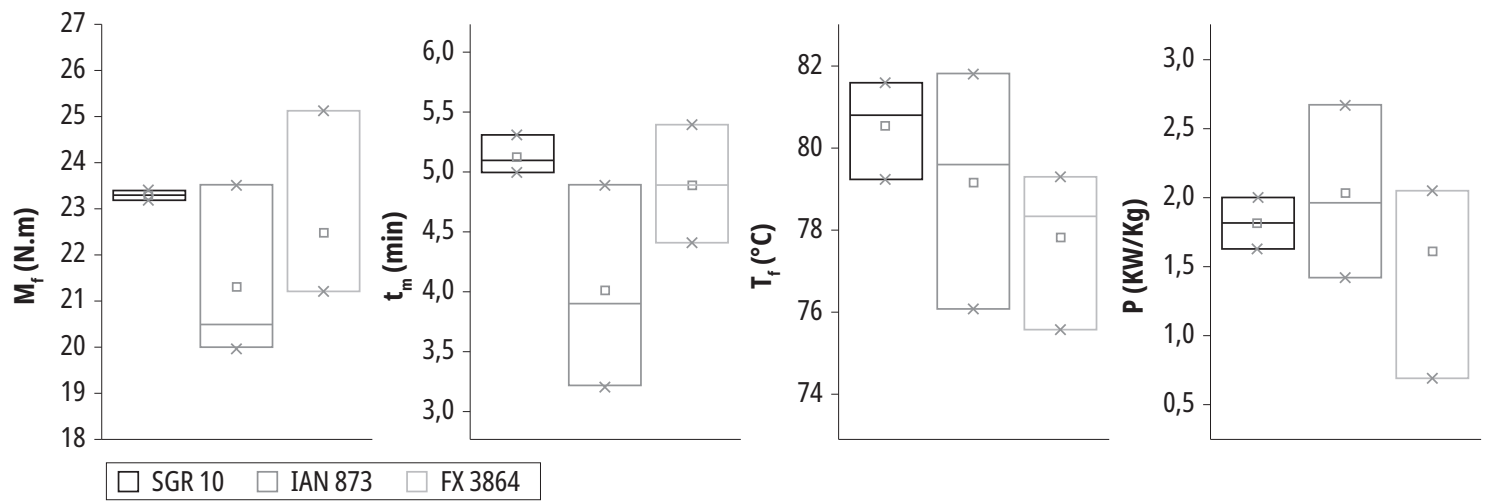

Figura 6. Diagrama de cajas para los valores de torque final, periodo de masticación y temperatura final. Fuente: Elaboración propia. 
clon IAN 873 requiere mayor potencia que el SGR 10 y el FX 3864. Estos resultados muestran que las diferencias respecto del contenido de gel entre los fondos de taza y el material de referencia no afectaron de manera significativa los parámetros de procesamiento en reómetro de torque, por lo que este material puede ser considerado como una alternativa para la producción de artículos con caucho natural. Sin embargo, es importante también considerar que las características de vulcanización son fundamentales para establecer los parámetros y las condiciones industriales, por tanto, debe observarse el comportamiento de cada compuesto al ser vulcanizado y así determinar la variabilidad en el contenido de gel que tiene un efecto en este comportamiento.

\section{Reometría de vulcanización}

En la figura 7, se presentan curvas representativas para las reometrías de vulcanización MDR a $160^{\circ} \mathrm{C}$ obtenidas para los compuestos elaborados a partir de los materiales objeto de estudio. Se observa que el torque de almacenamiento, asociado a la respuesta elástica S', para el SGR 10 presenta un comportamiento que tiende a ser lineal durante la etapa de inducción de la reacción de vulcanización, e inmediatamente, a medida que aumenta la densidad de reticulación, el valor de este parámetro se incrementa hasta alcanzar un valor máximo para posteriormente estabilizarse. En este punto de estabilidad, se considera que la reacción de vulcanización está completa [16]. No obstante, los compuestos elaborados a partir de los clones IAN 873 y FX 3864 presentaron reversión (disminución de S’) luego del periodo de estabilización. La reversión ocurre como consecuencia de la pérdida de la red entrecruzada que forman los enlaces de caucho natural con el azufre [17]. Esto puede ser una desventaja para el uso de fondos de taza de caucho natural, ya que este fenómeno afecta las propiedades mecánicas del producto final; sin embargo, existen aditivos empleados dentro de la formulación que pueden revertir este efecto además de permitir la vulcanización a temperaturas de hasta $175^{\circ} \mathrm{C}$ [17].

Al observar el comportamiento de la curva correspondiente a S", asociado a la respuesta viscosa, se logra apreciar que todas las curvas presentan al inicio una caída atribuible al acomodamiento entre los platos y la muestra por analizar, seguido de un aumento en que puede presentarse la formación de enlaces polisulfídicos pendientes entre las cadenas poliméricas del caucho y el acelerante durante la inducción de la reacción de vulcanización, lo que dificulta la movilidad de las cadenas y conlleva el incremento en su valor. A medida que la reacción de vulcanización avanza, el valor de S" tiende a estabilizarse para los compuestos elaborados a partir de los fondos de taza, mientras que para el SGR 10 se aprecia un aumento progresivo durante todo el ensayo. Esto indica que para el SGR 10 existe una
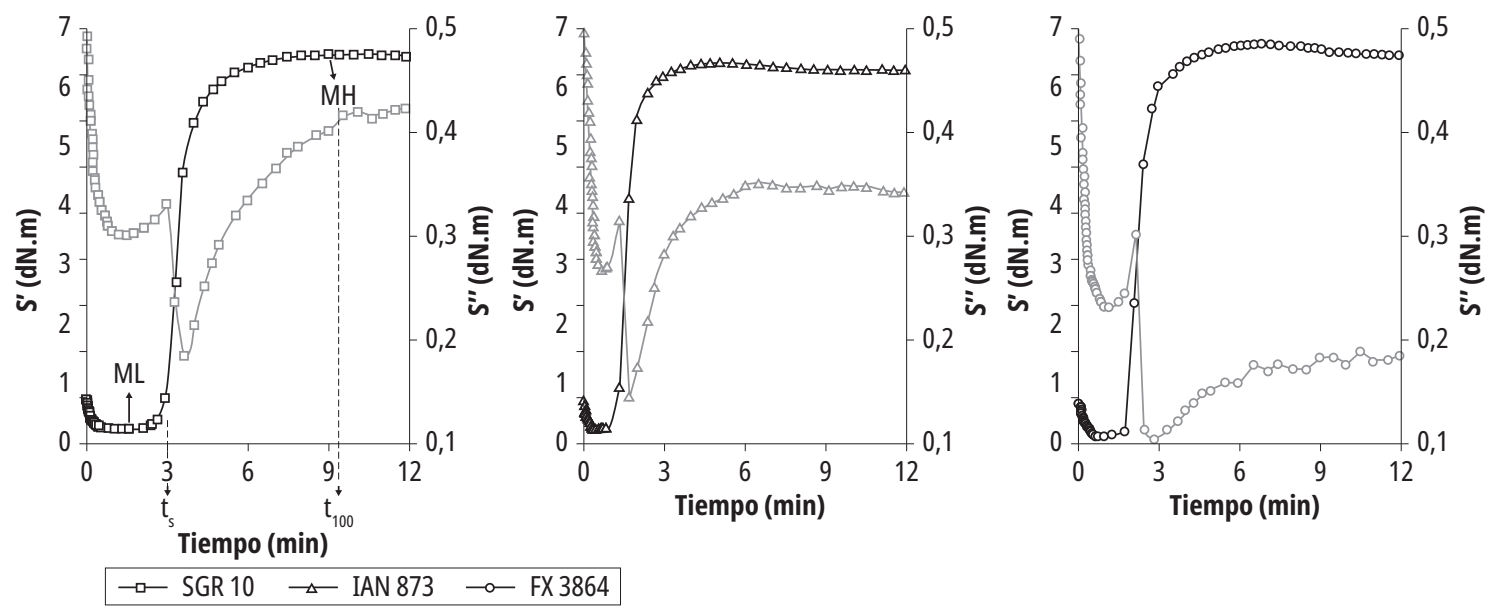

Figura 7. Curvas de reometría de vulcanización MDR representativas para las mezclas elaboradas a partir de SGR 10 y de fondos de taza de caucho natural de los clones IAN 873 y FX 3864 . Fuente: Elaboración propia. 
prevalencia de la respuesta viscosa durante la reacción de vulcanización [18]; esta respuesta puede estar fuertemente influenciada por la historia térmica que el material de referencia posee en el momento de ser evaluado.

En el tiempo scorch $\left(\mathrm{t}_{\mathrm{s}}\right)$ y el tiempo de vulcanización completa $\left(\mathrm{t}_{100}\right)$, se observa que los valores de los compuestos elaborados a partir de los fondos de taza son inferiores respecto del material de referencia SGR 10.

Las diferencias en los parámetros de vulcanización entre el material de referencia SGR 10 y los fondos de taza son atribuibles al hecho de que estos últimos presentan una proporción de elementos no caucho superior a la del SGR 10; entre estos elementos, las proteínas y fosfolípidos actúan como agentes naturales de aceleración de la reacción de vulcanización [19]. Sin embargo, en los compuestos elaborados a partir de fondos de taza, se observó reversión, lo que implica que dentro de la formulación deben emplearse aditivos que contrarresten este efecto y así garantizar las propiedades finales del compuesto [17].

En la figura 8, se presentan diagramas de caja para cada uno de los parámetros de vulcanización calculados a partir de las curvas de reometría MDR obtenidas a $160^{\circ} \mathrm{C}$.

En la figura 8, se observa que el compuesto que presenta mayor variabilidad en los valores de $\mathrm{MH}$ es el elaborado con los fondos de taza del clon IAN 873; sin embargo, tanto este como el del FX 3864 presentan un rango que abarca el presentado por el material de referencia SGR 10. En el ML, se aprecia que el material de referencia SGR 10 es el que presenta el mayor valor. Teniendo presente que este parámetro es una medida que facilita la evaluación del comportamiento del compuesto crudo, es decir, cuando no se ha dado inicio a la reacción de vulcanización, este es de gran utilidad como medida indirecta para la evaluación de la viscosidad, lo que indica que la mezcla elaborada con SGR 10 tiende a presentar una mayor oposición al flujo en crudo que los compuestos elaborados a partir de fondos de taza. A pesar de que la deformación aplicada durante la reometría MDR es pequeña comparada con la que está sometida al material al ser procesado en métodos convencionales como reometría de torque o molino abierto de rodillos, al ser una medida más cercana a la respuesta interna del material, puede relacionarse con lo observado durante los procesos de mezclado, en los que los fondos de taza presentaron una mayor facilidad para la masticación en molino abierto de rodillos, lo que es sumamente relevante a nivel industrial.

El comportamiento presentado por el $t_{s} y$ el $t_{100}$ se explica de acuerdo con las diferencias en el contenido de gel del material de referencia respecto de los fondos de taza, donde los compuestos no caucho que hacen parte de esta funcionan como acelerantes dentro de la reacción de vulcanización [19]. No obstante, se observa una diferencia significativa en el tiempo requerido para el inicio de la reacción de vulcanización, pero esta diferencia se acortó para el $t_{100}$, parámetro que resulta de gran
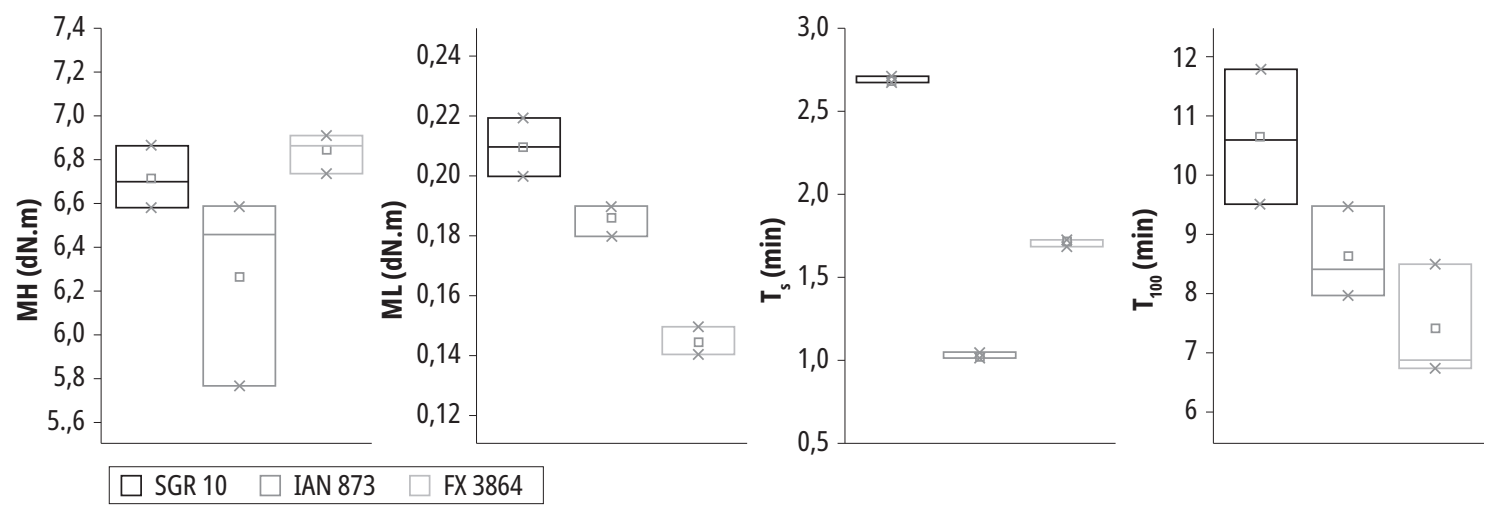

Figura 8. Parámetros de vulcanización obtenidos de las curvas de reometría MDR a $160^{\circ} \mathrm{C}$.

Fuente: Elaboración propia. 
importancia en el momento de la fabricación de productos en caucho, pues corresponde al tiempo total de vulcanización.

\section{Propiedades mecánicas en tensión y dureza Shore A}

$\mathrm{Al}$ obtener las láminas vulcanizadas a $160^{\circ} \mathrm{C}$ y troquelar las probetas de acuerdo con la geometría estipulada en la norma ASTM D412, se observó que el material de referencia SGR 10 presenta un color más claro respecto de las mezclas elaboradas a partir de los fondos de taza. Esto se atribuye a la presencia de compuestos polifenólicos que favorecen el oscurecimiento de los compuestos de caucho, encontrados en los compuestos no caucho que forman la fase gel en el caucho natural [20].
En la figura 9, se presentan curvas representativas de esfuerzo $(\mathrm{MPa})$ versus deformación unitaria (mm) para los compuestos evaluados, así como una ampliación de la zona comprendida entre 0 y 0,380 de deformación unitaria. Se observa que, para deformaciones de hasta 0,100 , el comportamiento de todas las muestras es igual, pero, a partir de 0,400, se aprecia una separación marcada entre el comportamiento de los compuestos elaborados con los fondos de taza y el material de referencia SGR 10, en que los esfuerzos involucrados para este son inferiores, lo que indica una menor rigidez.

En la figura 10, se muestran diagramas de caja para los valores de resistencia a la tensión, elongación a la rotura, módulos al 100 y al 300\% (M100 y M300) de deformación unitaria, que son medidas
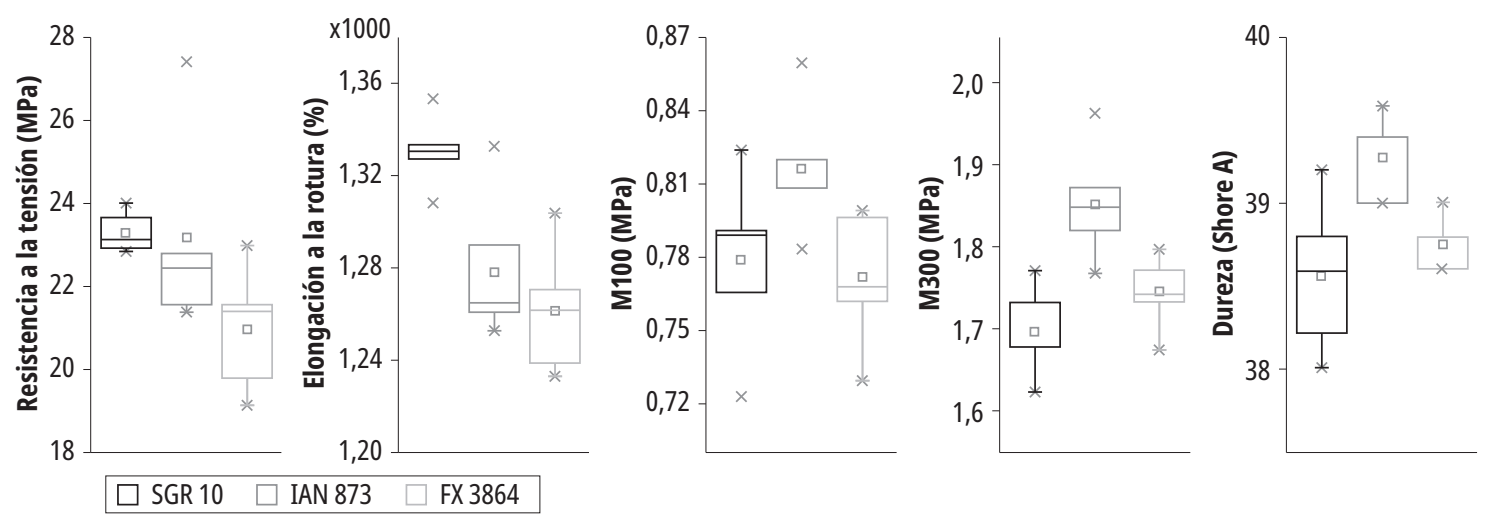

Figura 10. Propiedades en tensión y dureza Shore A obtenidas para los compuestos elaborados a partir de fondos de taza de caucho natural y SGR 10. Fuente: Elaboración propia.

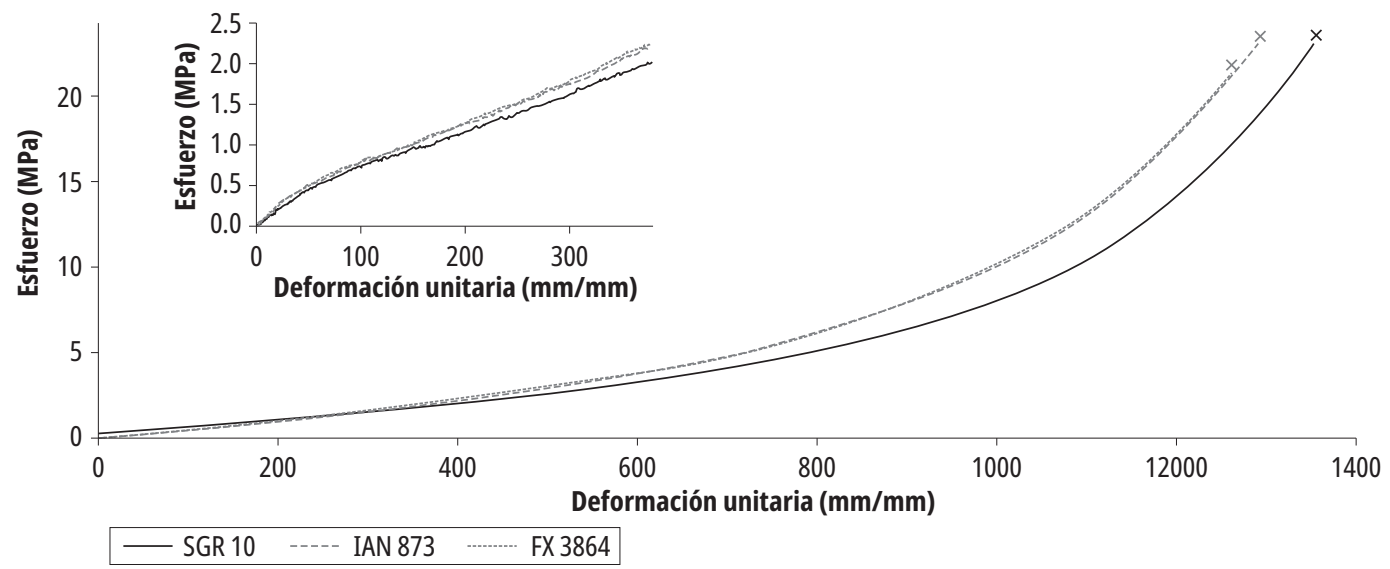

Figura 9. Curvas representativas de esfuerzo $(\mathrm{MPa})$ versus deformación unitaria $(\mathrm{mm} / \mathrm{mm})$ para las mezclas evaluadas. Fuente: Elaboración propia. 
comúnmente reportadas para la determinación de las propiedades tecnológicas de compuestos base caucho. De igual manera, se presentan los valores de dureza Shore A tomadas para cada material.

Se observa que el SGR es el material que presenta mayor resistencia a la tensión y elongación a la rotura; sin embargo, al efectuar un ANOVA con un 0,05 de significancia, fue posible determinar que, entre los materiales evaluados, solo existe una diferencia significativa para la elongación a la rotura. Al aplicar este mismo ANOVA, pero por pares, se encontró que existe una diferencia significativa en la resistencia a la tensión entre el FX 3864 y el SGR 10, así como para el M100 y el M300 entre el FX 3864 y el IAN 873. Esta información puede ser de utilidad para los fabricantes y usuarios de artículos en caucho según el comportamiento mecánico deseado según la aplicación.

$\mathrm{Al}$ observar el comportamiento de los valores M100 y M300, se aprecia una tendencia en que los compuestos elaborados a partir de los fondos de taza presentan una mayor rigidez que los obtenidos con el SGR 10, lo que es una ventaja para los fondos de taza, pues dentro de la formulación puede llegar a requerirse una menor cantidad de cargas reforzantes para alcanzar las propiedades mecánicas exigidas. En este sentido, es pertinente mencionar que existen estudios en los que se plantean relaciones entre el contenido de proteínas y las propiedades mecánicas, que demuestran que estos compuestos no caucho aumentan el módulo bajo tensión, debido a que actúan internamente como reforzantes en el compuesto vulcanizado [21, 22]; sin embargo, en este trabajo, no es posible determinar la existencia de una relación entre el comportamiento mecánico evidenciado por los fondos de taza y el contenido de nitrógeno determinado mediante análisis Kjeldahl o la mayor absorbancia presentada por las muestras en los picos asociados a los grupos funcionales a estos compuestos, ya que sería necesario garantizar que las muestras objeto de análisis sea el mismo en los ensayos de interés.

Por otra parte, es de anotar que el $\mathrm{MH}$ puede ser relacionado con la rigidez y la dureza del caucho vulcanizado; sin embargo, las diferencias entre las magnitudes de las deformaciones en tensión respecto de las aplicadas en un ensayo de reometría MDR no permiten efectuar una relación directa entre los resultados de ambas técnicas.

$\mathrm{Al}$ analizar todos los resultados de propiedades mecánicas y dureza, es posible establecer que, a pesar de que los compuestos elaborados a partir de los fondos de taza pueden presentar una mayor variabilidad, tienen propiedades tecnológicas que son similares a las obtenidas para el SGR 10.

\section{Conclusiones}

A partir de los resultados hallados en este estudio, fue posible determinar que las principales diferencias entre los fondos de taza de caucho natural y el SGR 10 es la mayor proporción de los elementos no caucho que presentan los fondos de taza, que, a pesar de mejorar la resistencia a la termooxidación, también inducen una mayor variabilidad. Los parámetros de procesamiento en reómetro de torque evidenciaron que no existen diferencias significativas entre estos materiales al ser expuestos a las mismas condiciones de velocidad y temperatura durante la masticación en este tipo de mezclador interno.

Los parámetros obtenidos mediante reometría de vulcanización permitieron establecer que existe una diferencia significativa en los parámetros ML, $t_{100} \mathrm{yt}_{\mathrm{s}}$, en que este último se pudo ver fuertemente influido por el efecto de los compuestos no caucho. En $\mathrm{MH}$ no se encontró diferencia significativa al comparar los materiales objeto de estudio.

$\mathrm{Al}$ evaluar las propiedades mecánicas en tensión y la dureza de los compuestos vulcanizados, fue posible evidenciar que el comportamiento de todos los compuestos es similar hasta deformaciones del 150\%. A partir de deformaciones del 400\%, se aprecia que el comportamiento de los compuestos elaborados a partir de los fondos de taza difiere respecto del material de referencia, por lo que se considera que el uso potencial de los fondos de taza puede ser más viable para productos que no estén expuestos a altas deformaciones en tensión. No se encontró diferencia entre las durezas de los materiales estudiados; sin embargo, debe 
considerarse que esto puede estar influido por el método manual empleado para la toma de dureza de los compuestos.

Los resultados obtenidos muestran que, a pesar de que los fondos de taza de caucho natural estudiados en este trabajo no fueron sometidos a procesos de estandarización, presentan propiedades tecnológicas similares e, incluso, en algunos casos superiores a las obtenidas por el caucho técnicamente especificado SGR 10. De esta manera, los fondos de taza constituyen una materia prima que puede ser una opción viable para la elaboración de piezas en caucho natural.

\section{Referencias}

[1] N. Arbeláez Mejía y A. Chejne Duarte, "Viabilidad comercial para la siembra de caucho y la extracción competitiva del látex en el Departamento de Córdoba”, tesis de grado, Colegio de Estudios Superiores de Administración, Bogotá, Colombia, 2017.

[2] N. T. Zapata Gallego, "Caracterización y propiedades reológicas de látex de caucho natural preservado proveniente de los clones IAN 710, IAN 873, FX 3864 y una mezcla entre los clones", tesis de maestría, Universidad Eafit, Medellín, Colombia, 2016.

[3] Fondo de Fomento Cauchero. (2016). Censo de plantaciones de caucho natural (Hevea brasiliensis) a año 2015 [En línea]. Disponible en: https://docs.wixstatic.com/ugd/e90b5c_dcbb611346314f53bd7ff8d4a$5 \mathrm{fbc} 2 \mathrm{c} 0 . \mathrm{pdf}$

[4] L. E. Forero Medina. (3 ag. 2017). Industria cauchera colombiana, gota a gota [En línea]. Disponible en: http://www.radiosantafe.com/2017/08/03/industria -cauchera-colombiana-gota-a-gota/

[5] S. Suchat, P. Theanjumpol y S. Karrila, "Rapid moisture determination for cup lump natural rubber by near infrared spectroscopy", Industrial Crops and Products, vol. 76, pp. 772-780, dic. 2015. https://doi.org/10.1016/j. indcrop.2015.07.070

[6] P. Nun-anan, S. Wisunthorn, S. Pichaiyut, N. Vennemann y C. Nakason, "Novel approach to determine non-rubber content in Hevea brasiliensis: influence of clone variation on properties of un-vulcanized natural rubber", Industrial Crops and Products, vol. 118, pp. 38-47, ag., 2018. https://doi.org/10.1016/j.indcrop. 2018.03.011

[7] T. Weber, M. G. Oliveira, M. Zeni, J. S. Crespo y R. C. Nunes, "Processability of revulcanizable SBR compositions”, Polymer Bulletin, vol. 61, no. 2, pp. 217-224, ag., 2008. DOI 10.1007/s00289-008-0937-3
[8] S. Thomas, C. H. Chan, L. A. Pothen, K. R. Rajisha y H. Maria, Eds., Natural rubber materials, vol. 1. Cambridge: Royal Society of Chemistry, 2013. http://dx.doi. org/10.1039/9781849737647-00073

[9] J. Carretero-González, T. A. Ezquerra, S. Amnuaypornsri, S. Toki, R. Verdejo, A. Sanz, J. Sakdapipanich, B. S. Hsiaod y M. A. López-Manchado, "Molecular dynamics of natural rubber as revealed by dielectric spectroscopy: The role of natural cross-linking", Soft Matter, vol. 6, no. 15, pp. 3636-3642, 2010. https://doi. org/10.1039/c003087b

[10] M. M. Rippel, C. A. Leite, L. T. Lee y F. Galembeck, "Direct imaging and elemental mapping of microgels in natural rubber particles", Colloid and Polymer Science, vol. 283, no. 5, pp. 570-574, febr. 2005. https:// doi.org/10.1007/s00396-004-1187-z

[11] B. Rodgers, ed., Rubber compounding: chemistry and applications, vol. 11. Boca Raton: CRC Press, 2016.

[12] M. N. Chukwu, J. A. Idiagha y M. O. Ihuezor, "Effect of acid coagulation level on the plasticity retention index (PRI) of natural rubber", Multidwasciplinary Journal of Research Development, vol. 15, no. 3, pp. 1-4, sept., 2010.

[13] M. S. Sambhi, "Degradative studies related to the plasticity retention index of the standard Malasyan rubber scheme-kinetics of degradation", Rubber Chemistry and Technology, vol. 55, no. 1, pp. 181-190, mzo., 1981. https://doi.org/10.5254/1.3535865

[14] S. Rolere, S. Liengprayoon, L. Vaysse, J. Sainte-Beuve y F. Bonfils, "Investigating natural rubber composition with Fourier Transform Infrared (FT-IR) spectroscopy: a rapid and non-destructive method to determine both protein and lipid contents simultaneously", Polymer Testing, vol. 43, pp. 83-93, my., 2015. https://doi. org/10.1016/j.polymertesting.2015.02.011

[15] A. M. Camacho Tamara, H. Reyes Pineda y A. Lozano Bohórquez, "Análisis y caracterización fisicoquímica del látex de caucho especie Hevea brasiliensis", Revista Tumbaga, vol. 9, no. 9, pp. 83-96, 2014.

[16] ASTM D5289, Standard Test Method for Rubber Property: Vulcanization Using Rotorless Cure Meters. ASTM, 2015.

[17] K. Bates, Enhancing the reversion resistance of natural rubber compounds [En línea]. Disponible en: http://digitaleditions.alsworthprintgroup.com/article/ Enhancing+the+reversion+resistance+of+natural+rubber+compounds/1558188/183199/article.html

[18] S. Mohammadian-Gezaz y M. Karrabi, "Characterization of the viscoelastic and vulcanization behavior of natural rubber nanocomposites having different levels 
of nano silicate/black", Progress in Rubber Plastics and Recycling Technology, vol. 33, no. 4, pp. 261-280, nov., 2017. https://doi.org/10.1177/147776061703300404

[19] W. Smitthipong, R. Tantatherdtam, K. Rungsanthien, P. Suwanruji, S. Klanarong, S. Radabutra y R. Chollakup, "Effect of non-rubber components on properties of sulphur crosslinked natural rubbers", Advanced Materials Research, vol. 844, pp. 345-348, 2013. https:// doi.org/10.4028/www.scientific.net/AMR.844.345

[20] S. Siriwong, A. Rungvichaniwat, P. Klinpituksa, K. H. Musa y A. Abdullah, "Color and antioxidant changes in various natural rubber processes", Applied Mechanics and Materials, vol. 754-755, pp. 230-234, febr. 2015. https://doi.org/10.4028/www.scientific.net/ AMM.754-755.230

[21] R. A. Rahim, A. Samsuri, D. Kamarun, A. Faiza y D. Veerasamy, "Tensile and tear strengths of post-vulcanised skim natural rubber latex films", Journal of Rubber Research, vol. 15, no. 4, pp. 243-254, en., 2016.

[22] O. Chaikumpollert, Y. Yamamoto y S. Kawahara, "Structural analysis of crosslinking junctions of vulcanized natural rubber by solid-state NMR spectroscopy equipped with field-gradient-magic angle spinning probe", KGK Kautschuk Gummi Kunststoffe, vol. 66, no. 7, pp. 28-33, 2013. 
